\title{
Nuclear magnetic resonance imaging of the heart Current status and future prospects
}

\author{
R E STEINER, G M BYDDER, A SELWYN, J DEANFIELD, D B LONGMORE, \\ R H KLIPSTEN, D FIRMIN
}

From the Departments of Diagnostic Radiology and Cardiology, Royal Postgraduate Medical School, Hammersmith Hospital, Du Cane Road, London; and National Heart Hospital, Westmoreland Street, London

SUMMARY The basic principles and current status of proton nuclear magnetic resonance imaging are outlined. Nuclear magnetic resonance images of the heart have improved in quality with advances in technique and better gating but clinical experience remains very limited. Myocardial infarction has been shown in animals and man, and plaques of atheroma have been seen with nuclear magnetic resonance flow studies.

Further improvements in image quality, faster examination times, ${ }^{23} \mathrm{Na}$ imaging, and the use of paramagnetic contrast agents are advances that are anticipated in the near future.

The medical application of nuclear magnetic resonance was pioneered by Odeblad, a Swedish physicist, who studied the properties of human tissues and fluids using proton spectroscopy during the 1950's and 1960's. ${ }^{2}$ Later workers showed abnormal proton nuclear magnetic resonance properties of animals' tumours in 1971 and $1972 .{ }^{34}$ The use of ${ }^{31} \mathrm{P}$ spectroscopy to study metabolism was first described in $1974^{5}$ and has been applied to the study of skeletal muscle, brain, and myocardium with considerable success. ${ }^{6}$

The essential concept for nuclear magnetic resonance imaging of applying a magnetic field gradient in order to locate the position of nuclei by changing their response frequence was described by Lauterbur in 1973.7 In vivo human proton heart images were first demonstrated in 1977 by Damadian et al.$^{8}$ Normal cardiac images have since been shown by other groups and technical advances including better cardiac gating have led to considerable improvement in image quality over the last two years.

The only other nucleus (apart from ${ }^{1} \mathrm{H}$ ) which has been successfully imaged is ${ }^{23} \mathrm{Na}$. Gated ${ }^{23} \mathrm{Na}$ images of a mouse heart were published in 1981.9 The low natural abundance of $31 \mathrm{P}$ in the body (one threethousandth part of that of hydrogen) places severe constraints on phosphorus imaging and further comment in this paper will relate only to proton imaging.

Accepted for publication 8 March 1983

\section{Instrumentation}

All nuclear magnetic resonance imaging machines are constructed around a large magnet which provides a uniform static magnetic field. Protons behave like tiny spinning magnets and this static magnetic field produces a net proton nuclear magnetisation in the patient (Fig. 1). Additional magnetic pulses oscillating at the spin frequency of the protons (several MegaHertz) are applied by means of a coil which surrounds the patient. These pulses rotate the nuclear magnetisation after which it relaxes or recovers back to its original position. For example, if a $90^{\circ}$ pulse is applied, the nuclear magnetisation is rotated from the longitudinal direction into the transverse plane (Fig. 2). After this rotation the component of the magnetisation in the long axis of the patient recovers from zero to its original amplitude in an exponential way. This recovery is called longitudinal or spin-lattice relaxation; it is approximately exponential and is characterised by the time constant $T_{1}$. Relaxation of the component of the magnetisation in the transverse direction back to its original amplitude of zero is termed transverse relaxation or spin-spin relaxation and is also approximately exponential. It is characterised by the time constant $T_{2}$. A receiver coil surrounds the patient and the electrical signal detected by this coil after such a $90^{\circ}$ pulse is known as the free 


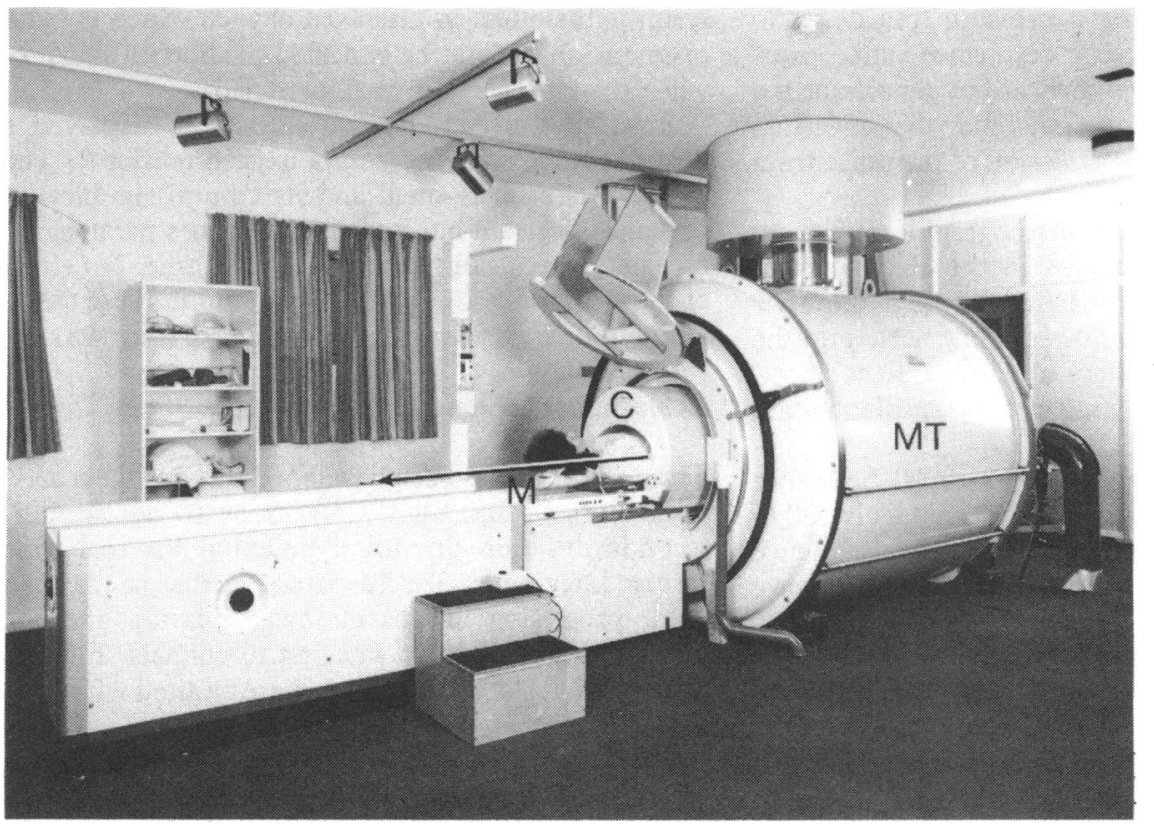

Fig. 1 A nuclear magnetic resonance scanner. The machine is constructed around a large cryomagnet (MT). The patient is slid into the bore of this machine and surrounded by a transmitter and receiver coil $(C)$. The magnet induces a net proton magnetisation $(M)$ in the patient.

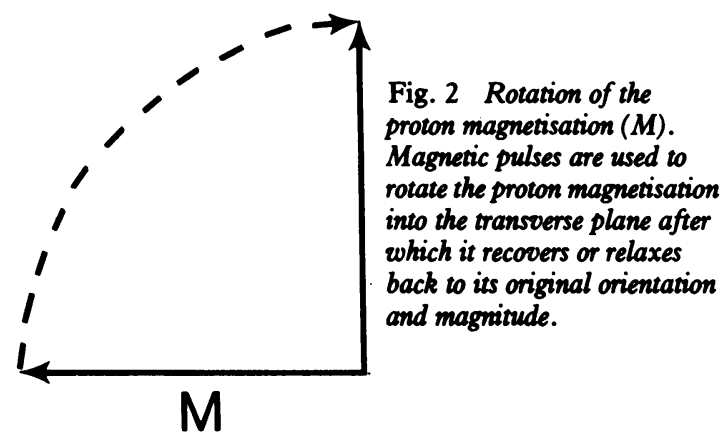

induction decay. It is this signal that is used to reconstruct the image.

Longitudinal relaxation $\left(T_{1}\right)$ depends on the interaction of protons with surrounding nuclei and molecules (the "lattice") while transverse relaxation $\left(T_{2}\right)$ depends on the interaction of protons with each other. Both $T_{1}$ and $T_{2}$ are sensitive indices of the local nuclear and molecular environment. By using a variety of pulse sequences it is possible to produce images with varying dependence on proton density $(\rho), T_{1}$ and $T_{2}$ (Table) The simplest of these is the repeated free induction decay sequence consisting of a succes-

Table Nuclear magnetic resonance pulse recordings

\begin{tabular}{ll}
\hline Pulse sequence & Principal image variables \\
\hline Steady state free precession & Proton density, $\mathrm{T}_{2} / \mathrm{T}_{1}$ \\
Repeated free induction decay & Proton density, $\mathrm{T}_{1}^{2}$ \\
Inversion recovery & Proton density, $\mathrm{T}_{1}^{1}$ \\
Spin echo & Proton density, $\mathrm{T}_{2}$ \\
\hline
\end{tabular}

sion of $90^{\circ}$ pulses. The resulting image largely reflects proton density with a small dependence on $T_{1}$.

The inversion recovery sequence begins with a $180^{\circ}$ pulse which inverts the direction of the magnetisation. The magnetisation is then allowed to recover for time $\tau$ after which a $90^{\circ}$ pulse is applied and the data are collected. The image depends on proton density but contrast is very dependent on changes in $T_{1}$.

The spin echo sequence involves the use of a $90^{\circ}$ pulse followed at time $\tau$ later by a $180^{\circ}$ pulse. The signal is collected a further time $\tau$ later. The contrast in these images is largely dependent on changes in $T_{2}$.

A more detailed account of imaging techniques has been published by Pykett et al.$^{10}$

\section{Cardiac gating}

After a pulse sequence, collection of nuclear magnetic resonance signal takes 10 to $30 \mathrm{~ms}$. A delay (typically of about $1000 \mathrm{~ms}$ ) is then necessary to allow the proton nuclear magnetisation to return to equilibrium. After this the pulse sequence is repeated. For the projection reconstruction technique the pulse sequence is typically repeated 180 times at $1^{\circ}$ intervals in the transverse plane in order to acquire sufficient data to reconstruct an image.

It is possible to produce cardiac images in which the data are collected irrespective of the phases of the cardiac cycle. This produces images that are largely diastolic because of the higher proportion of the cardiac cycle occupied by diastole.

Synchronisation of data collection to the peripheral 
pulse is achieved by using a pressure transducer to initiate the nuclear magnetic resonance pulse sequence. While this improves myocardial definition, the exact relation to cardiac events may be uncertain because of the delay in transmission of the pulse from the heart to the transducer.

The problems with electrocardiographic gating are the induced electrical currents in the electrocardiographic leads produced by magnetic field impulses and the introduction of radiofrequency noise into the system from outside. Filters are available to block outside interference, allowing precise cardiac gating timed in relation to the $R$ wave.

Over a pulse range of about 60 to 90 beats per minute the duration of systole is approximately 380 to $400 \mathrm{~ms}^{11}$ so that a typical inversion recovery sequence with the initial magnetic pulse triggered to the $R$ wave produces a late systolic or early diastolic scan (sampling is performed $400 \mathrm{~ms}$ later just after the $90^{\circ}$ pulse). An additional delay of 500 to $600 \mathrm{~ms}$ produces a later diastolic scan though the length of diastole is more variable than that of systole.

\section{Effect of oxygen on $\mathbf{T}_{1}$ of blood}

Initial human studies displayed a decrease in relaxation time $\left(T_{1}\right)$ of blood within the left ventricular cavity when subjects breathed $100 \%$ oxygen using ungated images. Studies of volunteers breathing $21 \%$, $40 \%, 60 \%, 80 \%$, and $100 \%$ oxygen showed a progressive decrease in $T_{1}$. A possible explanation for this

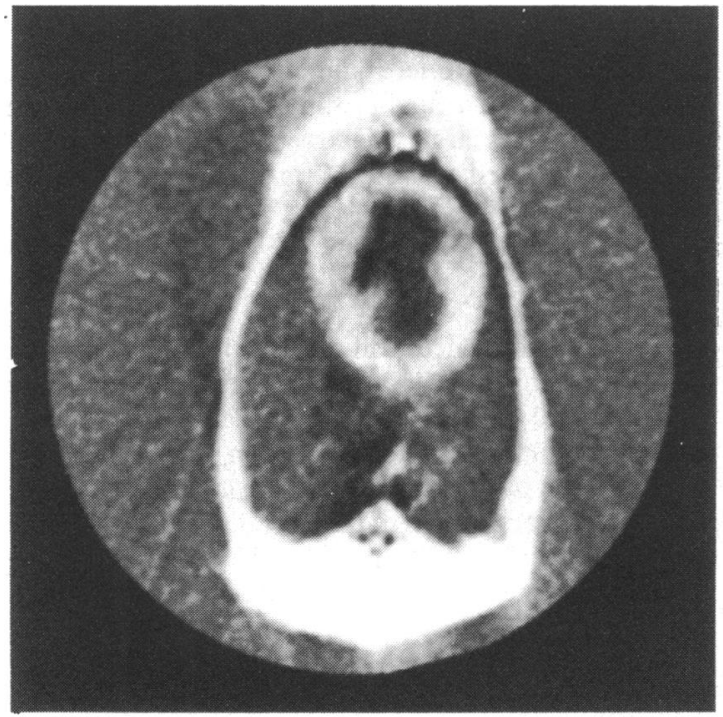

(A)

Fig. 3 Dog heart before $(A)$ and after $(B)$ occlusion of the left anterior descending artery: ungated inversion recovery scans. The left vig. 3 Dog heart before $(A)$ and after $(B)$ occlusion of the and cavity are well displayed in $(A)$. After occlusion of the left anterior descending artery a dark rim (long $\left.T_{1}\right)$ is seen around the anterolateral aspect of the myocardium. result is the effect of dissolved oxygen which is paramagnetic and would be expected to shorten blood $T_{1}$ when its concentration was increased.

Arterial blood studies on dogs also displayed a decrease in $T_{1}$ with increasing oxygen tension. ${ }^{12}$ The effect of oxygen is small and its clinical significance remains uncertain but it is of interest as a paramagnetic agent which can change relaxation time. No effect of oxygen on myocardial $T_{1}$ was found in these cases, unlike the results reported by Doyle et al. in 1981.13

\section{Echo-planar technique}

This technique has been developed by Ordidge et al. 14 It involves "single shot" images of 10 to 20 milliseconds duration providing potential for real time cardiac imaging. A $32 \times 32$ matrix size has so far been used, producing images of low resolution and the magnet size has limited imaging to animals. Further developments of this techinique are awaited with considerable interest.

\section{Experimental infarction}

Studies with proton spectroscopy in dogs after myocardial infarction have shown an increase in $T_{1}$ which correlates well with increase in water content. ${ }^{15} 16$

Using a steady state free precession technique (a pulse sequence which produces images dependent on $\rho$ and $\mathrm{T}_{2} / \mathrm{T}_{1}$ ) Buonanno et al.${ }^{17}$ were able to demon-

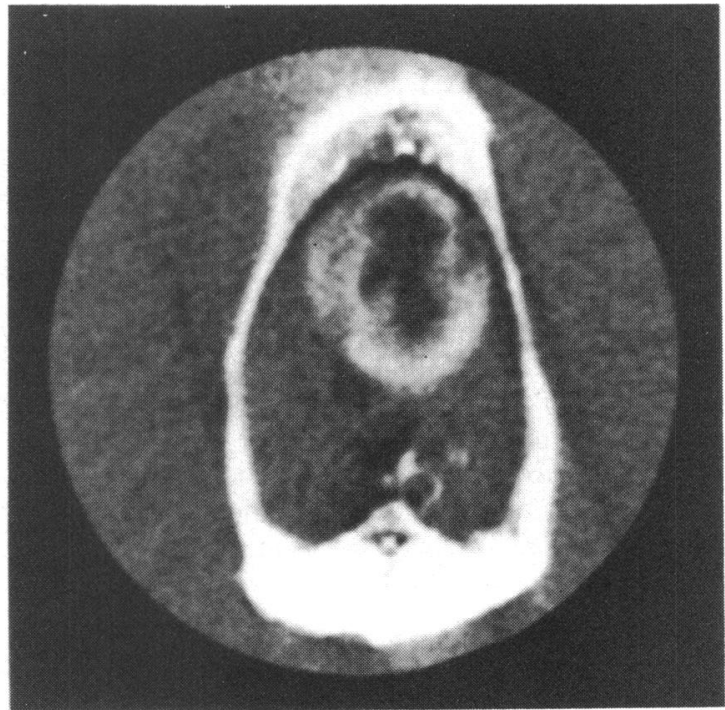

$(B)$ 


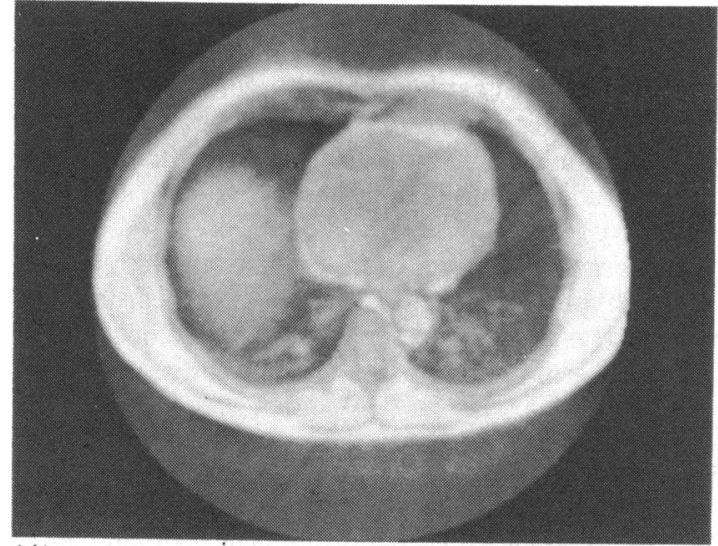

(A)

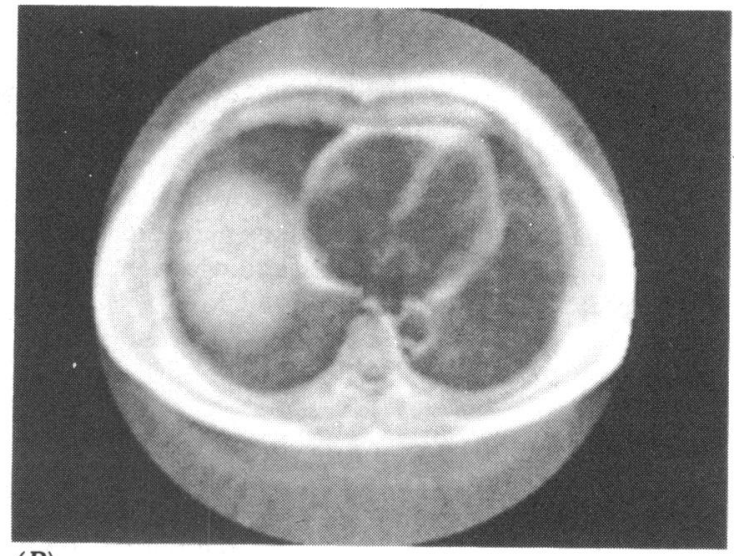

(B)

Fig. 4 Normal heart: ungated repeated free induction decay $(A)$ and inversion recovery $(B)$ scans. Little contrast is seen between blood and myocardium $(A)$ but they are clearly distinguished in $(B)$.

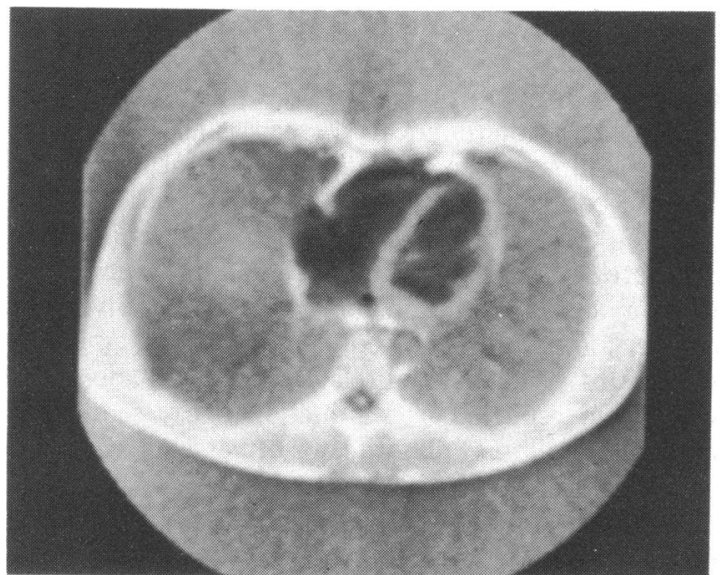

(A)

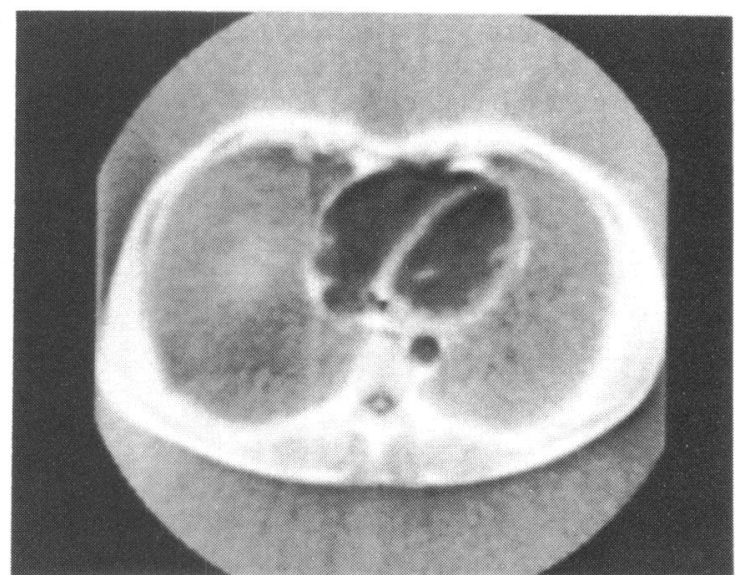

(B)

Fig. 5 Normal heart: pulse gated inversion recovery scans in systole $(A)$ and diastole $(B)$. Definition of the myocardium is better than in Fig. $4 B$ and the left ventricular cavity is seen to be larger in diastole.

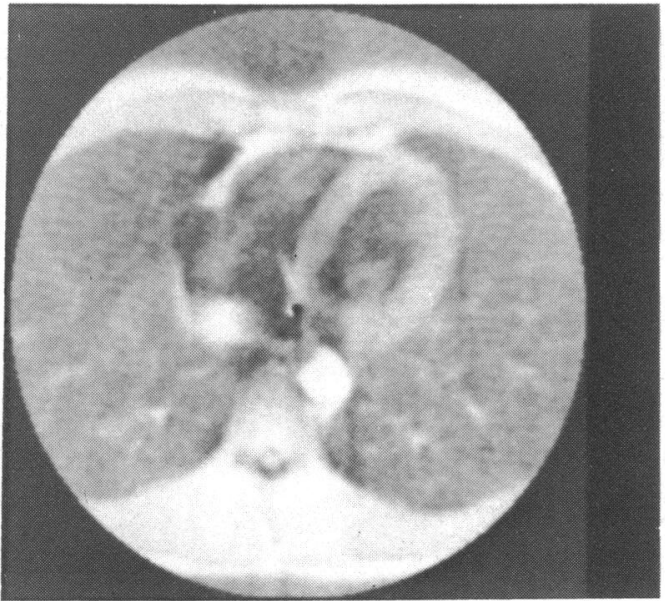

(A)

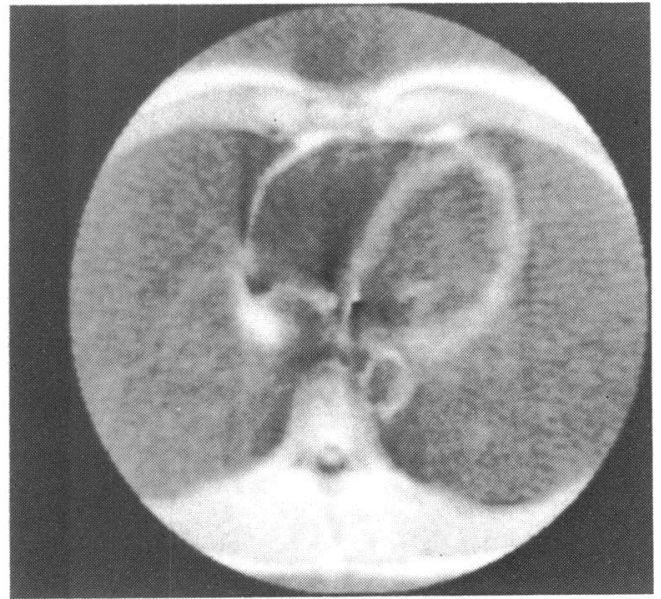

(B)

Fig. 6 Normal heart: electrocardiogram gated inversion recovery scans in systole $(A)$ and diastole $(B)$. A further improvement in myocardial definition is seen with electrocardiograph gating, and the difference between systole and diastole is readily seen. The blood in the aorta appears light in $(A)$ but dark in $(B)$. 
strate infarction in dogs, using manganese as a paramagnetic contrast agent; the infarct was not visible without it.

Using an ungated inversion recovery technique myocardial infarction has been shown in dogs after occlusion of the left anterior descending artery without the use of contrast agents (Fig. 3).

\section{Normal appearances}

Ungated human images have been produced by several investigators using techniques of selective excitation in which only the protons in the slice of interest are excited. ${ }^{18-21}$ Direct coronal and sagittal imaging is also possible.

Three dimensional techniques involving simultaneous collection of data from a volume have also been used and combined with cardiac gating. $22-24$

Repeated free induction decay images show little contrast between blood and myocardium but the distinction is readily seen with inversion recovery images (Fig. 4). The image quality is improved with pulse gating (Fig. 5) and electrocardiographic gating (Fig. 6). Valves and papillary muscles are readily seen.

Rapidly flowing blood in the aorta and inferior vena cava may appear light or dark, depending on the phase of the cardiac cycle.

\section{Clinical studies of heart}

These have been limited. Many of the nuclear magnetic resonance machines presently in use are situated in factories or other clinically disadvantageous sites and most of these machines are research prototypes designed to explore the possibilities of nuclear magnetic resonance rather than for use on a routine basis.

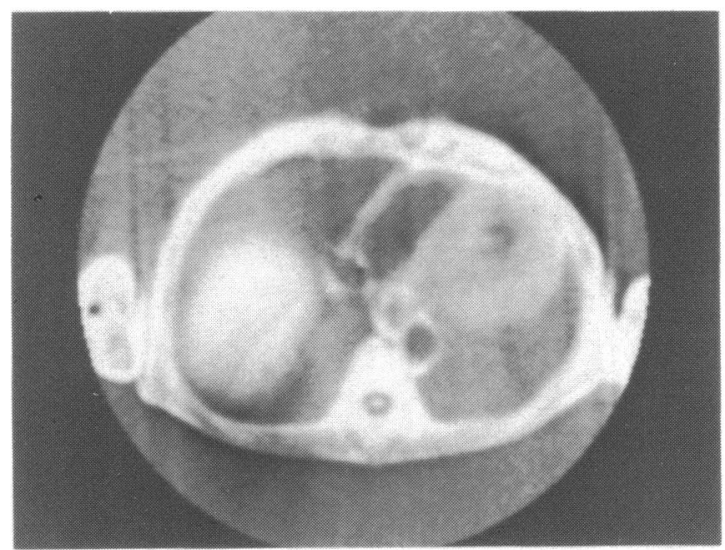

(A)

Fig. 7 Left ventricular hypertrophy (A) and hypertrophic obstructive cardiomyopathy (B): ungated inversion recovery scans. The enlarged ventricular wall and different contours of the ventricular cavities are evident.
Using electrocardiographic gated inversion recovery images, Go et al.$^{25}$ have shown evidence of old infarction and compared the results with dynamic isotope studies. Both techniques have shown a similar sensitivity.

Appearances arising from cardiac aneurysm and muscle hypertrophy (Fig. 7) have also been shown with nuclear magnetic resonance but its clinical application in cardiology remains virtually unexplored. ${ }^{26}$

\section{Vascular studies}

The flow dependence of the nuclear magnetic resonance signal has been recognised for a long time and attempts to produce a quantitative assessment of flow have been in progress for over 20 years. ${ }^{27}$ The flow dependence of the signal from the aorta in systole and diastole can be appreciated from Fig. 6 and flow profiles at different phases of the cardiac cycle can be produced. ${ }^{28}$ Atheroma can be seen as an obstruction to flow and may also display a high signal because of its lipid content. ${ }^{29}$

In the thorax there may be difficulty in distinguishing between vascular structures and tumours. The lack of signal from flowing blood using inversion recovery and spin echo sequences is very helpful in this regard. ${ }^{30}$ The lack of relative signal enhancement with rapid repeated free induction decay sequences may also be used as an indicator of vessel obstruction or occlusion.

It is also worth noting that exercise may produce obvious changes in the appearance of skeletal muscle. This is mainly an effect of increased blood flow to muscle $^{31}$ (Fig. 8), and may be relevant in the interpretation of $T_{1}$ changes in myocardium.

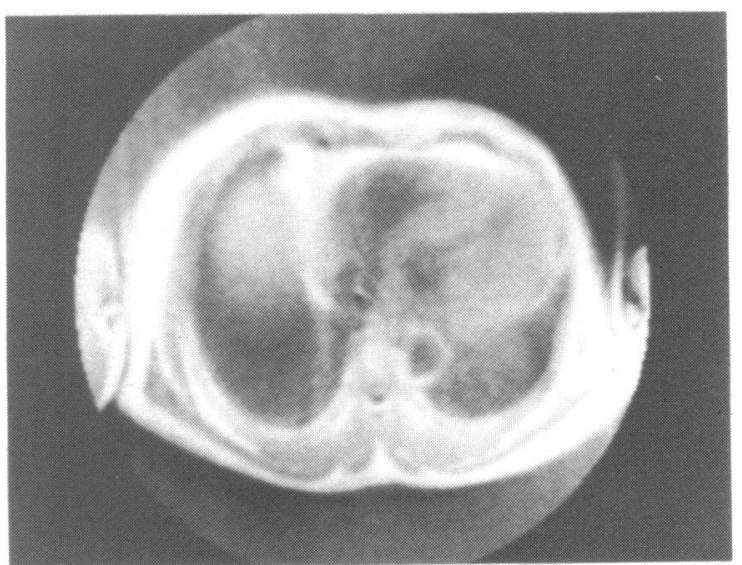

(B) 


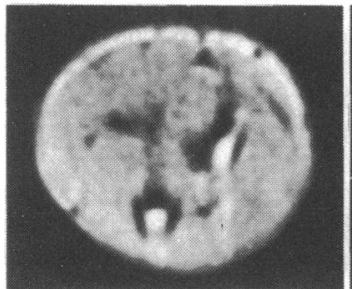

(A)

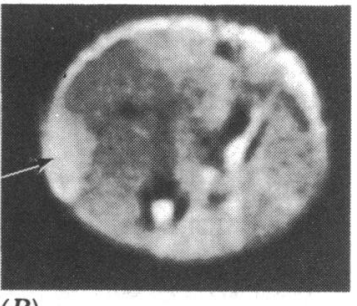

(B)

Fig. 8 Normal forearm: inversion recovery scans before $(A)$ and during $(B)$ exercise. After finger flexion the flexors of the fingers appear dark (long $T_{1}$ ). Flexor carpi ulnaris (arrow) is unchanged.

\section{Future developments}

Without doubt improvements in image quality will continue though probably not at the rate seen over the past two years.

The development of organic paramagnetic agents such as the nitroxide stable free radicles is an important advance. ${ }^{32}$ These so-called "spin labels" have been used in chemistry for many years. They appear to be non-toxic in animals and shorten relaxation time. They can be linked to metabolites or other substances of interest and appear to have considerable potential though it will take some time to assess toxicity before these agents can be considered for human use.

Much interest surrounds the possibility of obtaining clinically useful proton images and ${ }^{31} \mathrm{P}$ spectra on the same nuclear magnetic resonance machine. In order to obtain images the field must be low to avoid magnetic pulse absorption but for the production of spectra with good separation of peaks the field should be high. It is hoped that a compromise may be possible at a field strength of about 15 Kilogauss and work in this direction is proceeding at several centres.

${ }^{23} \mathrm{Na}$ images of the rat brain have been produced demonstrating brain infarction. These latter images displayed considerable contrast between normal and abnormal regions and the technique may have application in the human heart. ${ }^{33}$

With nuclear magnetic resonance still in a rapid phase of development assessment of its likely clinical role is not possible but the initial results are promising and this area of research is likely to be a source of considerable interest over the next few years.

We are grateful to the Department of Health and Social Security for their continuing support and to $\mathrm{Dr}$ Ian Young of Picker International who designed and built the nuclear magnetic resonance machine used to produce the above images.

\section{References}

1 Odeblad E, Lindström G. Some preliminary observations on the proton magnetic resonance in biologic samples. Acta Radiol 1955; 43: 469-76.

2 Odeblad E. Micro-NMR in high permanent magnetic fields. Acta Obstet Gynecol Scand 1966; suppl 2: 1-188.

3 Damadian R. Tumour detection by Nuclear Magnetic Resonance. Science 1971; 171: 1151-3.

4 Weisman ID, Bennett LH, Maxwell LR Sr, Woods MW, Burk D. Recognition of cancer in vivo by Nuclear Magnetic Resonance. Science 1972; 178: 1288-90.

5 Hoult DI, Busby SJW, Gadian DG, Radda GK, Richards RE, Seeley PJ. Observation of tissue metabolites using ${ }^{31} \mathrm{P}$ Nuclear Magnetic Resonance. Nature 1974; 252: 285-7.

6 Gadian DG, Radda GK. NMR studies of tissue metabolism. Annu Rev Biochem 1981; 50: 69-83.

7 Lauterbur PC. Image formation by induced local interactions: examples employing nuclear magnetic resonance. Nature 1973; 242: 190-1.

8 Damadian R, Goldsmith M, Minkoff L. NMR in cancer: XVI FONAR image of the live human body. Physiol Chem Phys 1977; 9: 97-100.

9 DeLayre JL, Ingwall JS, Malloy C, Fossel ET. Gated sodium-23 nuclear magnetic resonance images of an isolated perfused working rat heart. Science 1981; 212: 935-6.

10 Pykett IL, Newhouse JH, Buonanno FS, et al. Principles of nuclear magnetic resonance imaging. Radiology 1982; 143: 157-68.

11 Weissler AM, Garrard CL. Systolic time intervals in cardiac disease (I). Mod Concepts Cardiovasc Dis 1971; 15: $1-4$.

12 Bydder GM, Goatcher A, Hughes JMB, et al. Effect of oxygen tension on nuclear magnetic resonance spinlattice relaxation rate of blood in vivo [Abstract]. $\mathcal{F}$ Physiol (Lond) 1982; 332: 46P-7P.

13 Doyle FH, Gore JC, Penrock JM. Relaxation rate enhancement observed in vivo by NMR imaging. $f$ Comput Assist Tomogr 1981; 5: 295-6.

14 Ordidge RJ, Mansfield P, Coupland RE. Rapid biomedical imaging by NMR. Br f Radiol 1981; 54: 850-5.

15 Frank JA, Fales MA, House WV, et al. Measurement of proton nuclear magnetic longitudinal relaxation times and water content in infarcted canine myocardium and induced pulmonary injury. Clin Res 1976; 24: 217A.

16 Williams ES, Kaplan JI, Thatcher F, Zimmerman G, Knoebel SB. Prolongation of proton spin-lattice relaxation times in regionally ischemic tissue from dog hearts. f Nucl Med 1980; 21: 449-53.

17 Buonanno FS, Brady TJ, Pykett IL, et al. NMR clinical results: Massachusetts General Hospital. In: Partain CL, James AE, Rollo FD, Price RR, eds. NMR imaging. Philadelphia: Saunders, 1983: 207-30.

18 Hawkes RC, Holland GN, Moore WS, Roebuck EJ, Worthington BS. Nuclear magnetic resonance (NMR) tomography of the normal heart. $\mathcal{F}$ Comput Assist Tomogr 1981; 5: 605-12.

19 Edelstein WA, Hutchison JMS, Smith FW, Mallard JR, Johnson G, Redpath TW. Human whole body NMR tomographic imagings: normal sections. $B r f$ Radiol 1981; 54: 149-51. 
20 Young IR, Bailes DR, Bane $M$, et al. Initial clinical evaluation of a whole body nuclear magnetic resonance (NMR) tomograph. F Comput Assist Tomogr 1982; 6: 1-18.

21 Zeitler E, Schuierer E. NMR clinical results: Nuremburg. In: Partain CL, James AE, Rollo FD, Price RR, eds. NMR imaging. Philadelphia: Saunders, 1983: 26775.

22 Alfidi RJ, Haaga JR, El Yousef SJ, et al. Preliminary experimental results in human and animals with a superconducting, whole body nuclear magnetic resonance scanner. Radiology 1982; 143: 175-81.

23 Heidelberger E, Peterson SB, Lauterbur PC. 3D synchronised proton NMR imaging of the beating heart. $f$ Mag Res Med (In press).

24 Yeung HN, Kramer DM, Hunter WW, Hinshaw WS. Application of NMR imaging in cardiology. $f$ Mag Res Med (In press).

25 Go RT, MacIntyre WJ, Meaney TF, et al. Cardiac nuclear magnetic resonance imaging for visualisation of heart chambers myocardial and valvular structures and ventricular wall motion. $f$ Mag Res Med suppl 1 (In press).

26 Steiner RE. The Hammersmith clinical experience with nuclear magnetic resonance. Clin Radiol 1983; 34: 1323.

27 Singer JR. Blood flow measurements by NMR of the intact body. In: Partain CL, James AE, Rollo FD, Price RR, eds. NMR imaging. Philadelphia: Saunders, 1983: 168-78.
28 Macintyre WJ, Go RT, Cook JA, et al. Cardiac nuclear magnetic resonance imaging for visualisation of heart chambers myocardial and valvular structures and ventricular wall motion. Presented at the Radiological Society of North America Annual Meeting, 29 November 1982.

29 Kaufman L, Crooks LE, Sheldon PE, Rowan W, Miller T. Evaluation of NMR imaging for detection and quantification of obstruction in vessels. Invest Radiol 1982; 17: 554-60.

30 Welsh W, Gamsu G, Birnberg F, et al. NMR of the thorax in the diagnosis of bronchogenic carcinoma. $\mathcal{F}$ Mag Res Med suppl 1 (In press).

31 Young IR, Hall AS, Collins AG, Pennock JM, Spencer DH, Bydder GM. Some aspects of NMR in vivo imaging. Proc SPIE 1982; 347: 254-9.

32 Brasch RC, Nitecki DE, Lorden D, et al. Evaluation of nitroxide stable free radicals for contrast enhancement in NMR imaging. $f$ Mag Res Med suppl 1 (In press).

33 Hilal SK, Maudsley AA, Perman WH, Simon HE. Multinuclear NMR imaging in vivo. Presented at the Radiological Society of North America Annual Meeting, 29 November 1982.

Requests for reprints to Professor R E Steiner, Royal Postgraduate Medical School, Du Cane Road, London W12 0HS. 